\title{
Synchronizing linear systems via partial-state coupling *
}

\author{
S. Emre Tuna ${ }^{a}$ \\ ${ }^{a}$ Electrical and Electronics Engineering Department, Middle East Technical University, Ankara 06531, Turkey
}

\begin{abstract}
A basic result in synchronization of linear systems via output coupling is presented. For identical discrete-time linear systems that are detectable from their outputs and neutrally stable, it is shown that a linear output feedback law exists under which the coupled systems globally asymptotically synchronize for all fixed connected (asymmetrical) network topologies. An algorithm is provided to compute such feedback law based on individual system parameters.
\end{abstract}

Key words: multi-agent system; synchronization; consensus algorithm; partial-state coupling; output feedback.

\section{Introduction}

A notable meeting point for many researchers from different fields is the topic synchronization. One of the reasons for that comes from the nature as synchronization in large networks of dynamical systems is a frequently encountered phenomenon in biology. Among many others, one can count synchronously discharging neurons, crickets chirping in accord, and metabolic synchrony in yeast cell suspensions. Another reason is the abundance of technological applications: coupled synchronized lasers, vehicle formations, and sensor networks, just to name a few. We refer the reader to the surveys $[21,18,6,12]$ for references and more examples.

The main issue in studying the synchronization of coupled dynamical systems is the stability of synchronization. As in all cases where stability is the issue, the question whose answer is sought is Under what conditions will the individual systems synchronize? In a simplified yet widely-studied scenario, where the individual system dynamics are identical and the coupling between them is linear, studies focus on two ingredients: the dynamics of an individual system and the network topology. Start-

\footnotetext{
^ This paper was not presented at any IFAC meeting. The work was done when the author was a postdoctoral researcher at the University of Liege, Belgium. This paper presents research results of the Belgian Network DYSCO (Dynamical Systems, Control, and Optimization), funded by the Interuniversity Attraction Poles Programme, initiated by the Belgian State, Science Policy Office. The scientific responsibility rests with its author. Corresponding author S. E. Tuna. Tel. +90-312-210-2368.

Email address: sezaiemre@gmail.com (S. Emre Tuna).
}

ing with the agreement algorithm in [19] a number of contributions $[10,11,15,1,5,13]$ have gathered around the case where the weakest possible assumptions are made on the network topology at the expense of restrictive individual system dynamics. It was established in those works on multi-agent systems that when the individual system is taken to be an integrator and the coupling is of full-state, synchronization (consensus) results for timevarying interconnections whose unions ${ }^{1}$ over an interval are assumed to be connected instead of that each interconnection at every instant is connected.

Another school of research investigates networks with more complicated (nonlinear) individual system dynamics. When that is the case, the restrictions on the network topology have to be made stricter in order to ensure stability of synchronization. Generally speaking, more than mere connectedness of the network has been needed: coupling strength is required to be larger than some threshold and sometimes a symmetry ${ }^{2}$ or balancedness assumption is made on the connection graph. Different (though related) approaches have provided different insights over the years. The primary of such approaches is based on the calculations of the eigenvalues of the connection matrix and a parameter (e.g. the maximal Lyapunov exponent) depending on the individual system dynamics $[22,14]$. In endeavor to better understand synchronization stability, tools from systems theory such as Lyapunov functions [4,9], passivity $[3,17]$,

\footnotetext{
1 By union of interconnections we actually mean the union of the graphs representing the interconnections.

2 A network is symmetric if the matrix representing it is symmetric.
} 
contraction theory [16], and incremental input to state stability ( $\delta$ ISS) theory [8] have also proved useful.

This paper studies a broad class of linear systems under weak assumptions on the coupling structure and generalizes some of the existing results on synchronization. Namely, we consider identical individual discrete-time linear systems interacting via (diffusive) output coupling under a fixed (time-invariant) network topology. The contribution of the paper is in proving (via construction) the following basic result, which seems to have been missing from the literature. For a linear system ${ }^{3}$ that is neutrally stable and detectable from its output, there always exists a linear output feedback law that ensures the global asymptotic synchronization of any connected (not necessarily symmetric nor balanced) network of any number of coupled replicas of that system. To fortify our contribution practically, we provide an algorithm to compute one such feedback law. It is worth noting that our main theorem makes a compromise result between the two previously mentioned cases (i) where synchronization is established for very primitive individual system dynamics, such as that of an integrator, but under the weakest conditions on the network topology and (ii) where the network topology has to satisfy stronger conditions, such as that the coupling strength should be above a threshold, for want of achieving synchronization for nonlinear individual system dynamics.

The remainder of the paper is organized as follows. Notation and definitions reside in the next section. We give the problem statement along with our assumptions in Section 3. In Section 4 we provide a preliminary synchronization result on a network of linear systems with orthogonal system matrices. Then we generalize that result to establish our main theorem in Section 5.

\section{Notation and definitions}

The number of elements in a (finite) set $\mathcal{S}$ is denoted by $\# \mathcal{S}$. Let $\mathbb{N}$ denote the set of nonnegative integers. Let $|\cdot|$ denote 2 -norm. Identity matrix in $\mathbb{R}^{n \times n}$ is denoted by $I_{n}$. A matrix $Q \in \mathbb{R}^{n \times n}$ is orthogonal if $Q Q^{T}=$ $Q^{T} Q=I_{n}$. Orthogonal matrices satisfy $|Q v|=|v|$ for all $v \in \mathbb{R}^{n}$. Given $C \in \mathbb{R}^{m \times n}$ and $A \in \mathbb{R}^{n \times n}$, pair $(C, A)$ is observable if $\left[C^{T} A^{T} C^{T} A^{2 T} C^{T} \ldots A^{(n-1) T} C^{T}\right]$ is full row rank. Pair $(C, A)$ is detectable (in the discrete-time sense) if that $C A^{k} x=0$ for some $x \in \mathbb{R}^{n}$ and for all $k \in \mathbb{N}$ implies $\lim _{k \rightarrow \infty} A^{k} x=0$. Matrix $A \in \mathbb{R}^{n \times n}$ is neutrally stable (in the discrete-time sense) if it has no eigenvalue with magnitude greater than unity and the Jordan block corresponding to an eigenvalue $\lambda$ with $|\lambda|=1$ is of size one. ${ }^{4}$ Let $\mathbf{1} \in \mathbb{R}^{p}$ denote the vector with all entries equal to unity.

4 Note that $A$ is neutrally stable iff there exists a symmetric positive definite matrix $P$ such that $A^{T} P A-P \leq 0,[2]$.
}

Kronecker product of $A \in \mathbb{R}^{m \times n}$ and $B \in \mathbb{R}^{p \times q}$ is

$A \otimes B:=\left[\begin{array}{ccc}a_{11} B & \cdots & a_{1 n} B \\ \vdots & \ddots & \vdots \\ a_{m 1} B & \cdots & a_{m n} B\end{array}\right]$

In the pages to come we will enjoy the properties $(A \otimes$ $B)(C \otimes D)=(A C) \otimes(B D)$ (provided that products $A C$ and $B D$ are allowed), $A \otimes B+A \otimes C=A \otimes(B+C$ ) (for $B$ and $C$ that are of same size) and $|A \otimes B|=|A||B|$.

Matrix $P \in \mathbb{R}^{n \times n}$ is an orthogonal projection onto the subspace range $(P)$ if $P^{2}=P$ and $P^{T}=P$. For an orthogonal projection $P$, if the columns of $C^{T} \in \mathbb{R}^{n \times m}$ are an orthonormal basis for range $(P)$ then $P=C^{T} C$. Matrix $V=I_{n}-P$ is also an orthogonal projection and $\operatorname{range}(V)=\operatorname{range}(P)^{\perp}$. It is easy to see that $P V=$ $V P=0$.

A (directed) graph is a pair $(\mathcal{N}, \mathcal{A})$ where $\mathcal{N}$ is a nonempty finite set (of nodes) and $\mathcal{A}$ is a finite collection of pairs $(\operatorname{arcs})\left(n_{i}, n_{j}\right)$ with $n_{i}, n_{j} \in \mathcal{N}$. A path from $n_{1}$ to $n_{\ell}$ is a sequence of nodes $\left\{n_{1}, \ldots, n_{\ell}\right\}$ such that $\left(n_{i}, n_{i+1}\right)$ is an arc for $i \in\{1, \ldots, \ell-1\}$. A graph is connected if it has a node to which there exists a path from every other node. ${ }^{5}$

The graph of a matrix $\Lambda:=\left[\lambda_{i j}\right] \in \mathbb{R}^{p \times p}$ is the pair $(\mathcal{N}, \mathcal{A})$ where $\mathcal{N}=\left\{n_{1}, \ldots, n_{p}\right\}$ and $\left(n_{i}, n_{j}\right) \in \mathcal{A}$ iff $\lambda_{i j}>0$. Matrix $\Lambda$ is said to be connected (in the discretetime sense) if it satisfies:

(i) $\lambda_{i i}>0$ and $\lambda_{i j} \geq 0$ for all $i, j$;

(ii) each row sum equals 1 ;

(iii) its graph is connected. ${ }^{6}$

For $\Lambda$ that is connected, it is known that $\lim _{k \rightarrow \infty} \Lambda^{k}=$ $\mathbf{1} r^{T}$ where $r \in \mathbb{R}^{p}$ has nonnegative entries and satisfies $r^{T} \mathbf{1}=1$. We mention that, in an interconnection of systems, if the matrix describing the network topology satisfies properties (i) and (ii) above, then the coupling between the systems is said to be diffusive.

Given maps $\xi_{i}: \mathbb{N} \rightarrow \mathbb{R}^{n}$ for $i \in\{1, \ldots, p\}$ and a map $\bar{\xi}: \mathbb{N} \rightarrow \mathbb{R}^{n}$, the elements of the set $\left\{\xi_{i}(\cdot): i=1, \ldots, p\right\}$ are said to synchronize to $\bar{\xi}(\cdot)$ if $\left|\xi_{i}(k)-\bar{\xi}(k)\right| \rightarrow 0$ as $k \rightarrow \infty$ for all $i$.

\footnotetext{
${ }^{5}$ Note that this definition of connectedness for directed graphs is weaker than strong connectivity and stronger than weak connectivity.

${ }^{6}$ Recall that for continuous time applications, definition of connectedness is different: a matrix $\left[\gamma_{i j}\right]$ is considered connected (in the continuous-time sense) if $\gamma_{i j} \geq 0$ for $i \neq j$; each row sum equals 0; and its graph is connected.
} 


\section{Problem statement}

\subsection{Systems under study}

We consider $p$ identical discrete-time linear systems

$x_{i}^{+}=A x_{i}+u_{i}, \quad y_{i}=C x_{i}, \quad i=1, \ldots, p$

where $x_{i} \in \mathbb{R}^{n}$ is the state, $x_{i}^{+}$is the state at the next time instant, $u_{i} \in \mathbb{R}^{n}$ is the input, and $y_{i} \in \mathbb{R}^{m}$ is the output of the $i$ th system. Matrices $A$ and $C$ are of proper dimensions. The solution of $i$ th system at time $k \in \mathbb{N}$ is denoted $x_{i}(k)$. In this paper we consider the case where at each time instant only the following information

$z_{i}=\sum_{j=1}^{p} \lambda_{i j}\left(y_{j}-y_{i}\right)$

is available to $i$ th system to determine an input value where $\Lambda:=\left[\lambda_{i j}\right] \in \mathbb{R}^{p \times p}$ is the matrix describing the network topology. Matrix $\Lambda$ has nonnegative entries, strictly positive diagonal entries, and rows summing up to one. That is, the coupling between systems is diffusive.

\subsection{Assumptions made}

We make the following assumptions on systems (1) which will henceforth hold.

(A1) $A$ is neutrally stable.

(A2) $(C, A)$ is detectable.

\subsection{Objectives}

We have two objectives in this paper. The first one is to find the answer to the question Does there exist a linear feedback law $L \in \mathbb{R}^{n \times m}$ such that solutions of systems (1) with $u_{i}=L z_{i}$, where $z_{i}$ is as in (2), globally synchronize for all connected $\Lambda$ ? Our second objective is, if the answer to the previous question is affirmative, to devise an algorithm to compute one such $L$.

\section{A preliminary result}

Consider $p$ interconnected systems

$\xi_{i}^{+}=Q \xi_{i}+Q H^{T} H \sum_{j=1}^{p} \lambda_{i j}\left(\xi_{j}-\xi_{i}\right), \quad i=1, \ldots, p$

with $Q \in \mathbb{R}^{n \times n}$ and $H \in \mathbb{R}^{m \times n}$. We make the following assumptions on systems (3) which will henceforth hold.

(B1) $Q$ is orthogonal.

(B2) $H H^{T}=I_{m}$.

(B3) $(H, Q)$ is observable.
The following result can be implicitly found, for instance, in [20, Cor. 15].

Lemma 1 Given $\Lambda \in \mathbb{R}^{p \times p}$ that is connected, let $r \in \mathbb{R}^{p}$ be such that $\lim _{k \rightarrow \infty} \Lambda^{k}=\mathbf{1} r^{T}$. Then there exist $c \geq 1$ and $\sigma \in(0,1)$ such that $\left|\Lambda^{k}-\mathbf{1} r^{T}\right| \leq$ c $\sigma^{k}$ for all $k \in \mathbb{N}$.

We also need the following result for later use.

Lemma 2 Pair $(H, Q)$ of (3) satisfies

$\left|\prod_{i=0}^{n-1}\left(I_{n}-Q^{i T} H^{T} H Q^{i}\right)\right|<1$.

PROOF. Note that $Q^{i T} H^{T} H Q^{i}$ is an orthogonal projection for $i=0, \ldots, n-1$. Whence $\left|I_{n}-Q^{i T} H^{T} H Q^{i}\right| \in$ $\{0,1\}$. Now, suppose (4) is not true. Then there exists $v \in \mathbb{R}^{n}$ with $|v|=1$ such that

$\left|\left(I_{n}-Q^{(n-1) T} H^{T} H Q^{n-1}\right) \cdots\left(I_{n}-H^{T} H\right) v\right|=1$.

For $w \in \mathbb{R}^{n}$ and an orthogonal projection $P \in \mathbb{R}^{n \times n}$, if $\left(I_{n}-P\right) w \neq w$ then it must be that $\left|\left(I_{n}-P\right) w\right|<|w|$. As a consequence we must have $Q^{i T} H^{T} H Q^{i} v=0$ for all $i$. Thence $H Q^{i} v=0$ for all $i$. This means that $v$ is orthogonal to every column vector of $\left[H^{T} Q^{T} H^{T} Q^{2 T} H^{T} \ldots Q^{(n-1) T} H^{T}\right]$. This however is a contradiction for $(H, Q)$ pair is observable.

We now provide a key result.

Theorem 3 Consider systems (3). Suppose $\Lambda$ is connected and let $r \in \mathbb{R}^{p}$ be such that $\lim _{k \rightarrow \infty} \Lambda^{k}=\mathbf{1} r^{T}$. Then solutions $\xi_{i}(\cdot)$ for $i=1, \ldots$, p synchronize to

$\bar{\xi}(k):=\left(r^{T} \otimes Q^{k}\right)\left[\begin{array}{c}\xi_{1}(0) \\ \vdots \\ \xi_{p}(0)\end{array}\right]$

PROOF. Let us stack individual system states to obtain $\mathbf{x}:=\left[\begin{array}{lll}\xi_{1}^{T} & \ldots & \xi_{p}^{T}\end{array}\right]^{T}$. From (3) we obtain

$\mathbf{x}^{+}=\left(I_{p} \otimes Q\right)\left(I_{p} \otimes I_{n}+\left(\Lambda-I_{p}\right) \otimes\left(H^{T} H\right)\right) \mathbf{x}$.

Let $\mathbf{w}(k):=\left(I_{p} \otimes Q^{-k}\right) \mathbf{x}(k), P_{k}:=Q^{k T} H^{T} H Q^{k}$, and $V_{k}:=I_{n}-P_{k}$ for $k \in \mathbb{N}$. Observe that for each $k, P_{k}$ is an orthogonal projection onto subspace range $\left(Q^{k T} H^{T}\right)$ and $V_{k}$ onto range $\left(Q^{k T} H^{T}\right)^{\perp}$. From (5) we can write

$\mathbf{w}(k+1)=\left(I_{p} \otimes V_{k}+\Lambda \otimes P_{k}\right) \mathbf{w}(k)$. 
For $k, h \in \mathbb{N}$ with $k \geq h$ Let

$\Phi(k, h):=\prod_{\tau=h}^{k-1}\left(I_{p} \otimes V_{\tau}+\Lambda \otimes P_{\tau}\right)$

with $\Phi(h, h)=I_{p n}$. Note that $\mathbf{w}(k)=\Phi(k, h) \mathbf{w}(h)$. We now establish the following:

$\lim _{k \rightarrow \infty} \Phi(k, h)=\mathbf{1} r^{T} \otimes I_{n}$

for any fixed $h$. Without loss of generality let $h=0$. For $\ell, k \in \mathbb{N}$ with $\ell \leq k$ let us define

$M_{\ell, k}:=\sum_{M \in \Omega_{\ell, k}} M$

where

$$
\begin{aligned}
& \Omega_{\ell, k}:=\left\{M: M=L_{k-1} L_{k-2} \cdots L_{0},\right. \\
&\left.L_{i} \in\left\{V_{i}, P_{i}\right\}, \#\left\{i: L_{i}=P_{i}\right\}=\ell\right\} .
\end{aligned}
$$

For instance, $\Omega_{0,4}=\left\{V_{3} V_{2} V_{1} V_{0}\right\}$ and $\Omega_{2,4}=\left\{V_{3} V_{2} P_{1} P_{0}\right.$, $\left.V_{3} P_{2} V_{1} P_{0}, P_{3} V_{2} V_{1} P_{0}, V_{3} P_{2} P_{1} V_{0}, P_{3} V_{2} P_{1} V_{0}, P_{3} P_{2} V_{1} V_{0}\right\}$. Observe that

$\# \Omega_{\ell, k}=\frac{k !}{(k-\ell) ! \ell !}=:\left(\begin{array}{l}k \\ \ell\end{array}\right)$

Note that $M_{\ell, k+1}=V_{k} M_{\ell, k}+P_{k} M_{\ell-1, k}$ for $\ell \in$ $\{1, \ldots, k\}, M_{0, k+1}=V_{k} M_{0, k}$, and $M_{k+1, k+1}=P_{k} M_{k, k}$. We can write

$\Phi(k, 0)=\sum_{\ell=0}^{k}\left(\Lambda^{\ell} \otimes M_{\ell, k}\right)$

and

$\sum_{\ell=0}^{k} M_{\ell, k}=I_{n}$

Let $\alpha:=\left|V_{n-1} V_{n-2} \cdots V_{0}\right|$. Lemma 2 guarantees that $\alpha<1$. We make the following observations. For all $\ell, k$,

$\left|M_{\ell, k}\right| \leq 1$

and

$\left|M_{\ell, k}\right| \leq\left(\begin{array}{l}k \\ \ell\end{array}\right) \alpha^{\left\lfloor\frac{k+1}{n}\right\rfloor-\ell}$

for $\lfloor(k+1) / n\rfloor-\ell \geq 0$. Let us first show (12). Suppose for some $k \in \mathbb{N}$ and all $v \in \mathbb{R}^{n}$ with $|v|=1$ we have

$\sum_{\ell=0}^{k}\left|M_{\ell, k} v\right|^{2}=1$
Then we can write, since both $V_{k}$ and $P_{k}$ are orthogonal projections satisfying $V_{k} P_{k}=0$,

$$
\begin{aligned}
\sum_{\ell=0}^{k+1}\left|M_{\ell, k+1} v\right|^{2}= & \left|V_{k} M_{0, k} v\right|^{2}+\left|P_{k} M_{k, k} v\right|^{2} \\
& +\sum_{\ell=1}^{k}\left|V_{k} M_{\ell, k} v+P_{k} M_{\ell-1, k} v\right|^{2} \\
= & \left|V_{k} M_{0, k} v\right|^{2}+\left|P_{k} M_{k, k} v\right|^{2} \\
& +\sum_{\ell=1}^{k}\left(\left|V_{k} M_{\ell, k} v\right|^{2}+\left|P_{k} M_{\ell-1, k} v\right|^{2}\right) \\
= & \sum_{\ell=0}^{k}\left(\left|V_{k} M_{\ell, k} v\right|^{2}+\left|P_{k} M_{\ell, k} v\right|^{2}\right) \\
= & \sum_{\ell=0}^{k}\left|M_{\ell, k} v\right|^{2} \\
= & 1 .
\end{aligned}
$$

Hence, by induction, (14) holds for all $k$ since it trivially holds for $k=0$ thanks to (11). We therefore have (12) as a direct implication of (14).

Now, we show (13). Let us be given some $k \geq n-1$. Let then $k_{0}:=k-n+1$. We observe that

$$
\begin{aligned}
& \left|V_{k} V_{k-1} \cdots V_{k-n+1}\right| \\
& \quad=\left|Q^{k_{0} T} V_{n-1} Q^{k_{0}} Q^{k_{0} T} V_{n-2} Q^{k_{0}} \cdots Q^{k_{0} T} V_{0} Q^{k_{0}}\right| \\
& \quad=\left|Q^{k_{0} T} V_{n-1} V_{n-2} \cdots V_{0} Q^{k_{0}}\right| \\
& \quad=\left|V_{n-1} V_{n-2} \cdots V_{0}\right| \\
& \quad=\alpha
\end{aligned}
$$

Hence for $M \in \Omega_{\ell, k}$ one can write

$|M| \leq \alpha^{\left\lfloor\frac{k+1}{n}\right\rfloor-\ell}$

for $\lfloor(k+1) / n\rfloor-\ell \geq 0$. Then by (8) and (9) we obtain (13).

Now we are ready to show (7). Let us be given $\delta>0$. Let $c \geq 1$ and $\sigma \in(0,1)$ be such that $\left|\Lambda^{k}-\mathbf{1} r^{T}\right| \leq c \sigma^{k}$ for all $k \in \mathbb{N}$. Such $c$ and $\sigma$ exist by Lemma 1 . Choose $\ell^{*} \in \mathbb{N}$ such that $\sum_{\ell=\ell^{*}}^{\infty} c \sigma^{\ell} \leq \delta / 2$. Then choose $k^{*} \in \mathbb{N}$ such that

$\sum_{\ell=0}^{\ell^{*}-1}\left(\begin{array}{l}k \\ \ell\end{array}\right) \alpha^{\left\lfloor\frac{k+1}{n}\right\rfloor-\ell} \leq \frac{\delta}{2 c}$

for all $k \geq k^{*}$. Now, let us be given some $k \geq k^{*}$. We 
write by (10) and (11)

$$
\begin{aligned}
& \left|\Phi(k, 0)-\mathbf{1} r^{T} \otimes I_{n}\right| \\
& =\left|\sum_{\ell=0}^{k} \Lambda^{\ell} \otimes M_{\ell, k}-\sum_{\ell=0}^{k} \mathbf{1} r^{T} \otimes M_{\ell, k}\right| \\
& =\left|\sum_{\ell=0}^{k}\left(\Lambda^{\ell}-\mathbf{1} r^{T}\right) \otimes M_{\ell, k}\right| \\
& \leq \sum_{\ell=0}^{\ell^{*}-1}\left|\left(\Lambda^{\ell}-\mathbf{1} r^{T}\right) \otimes M_{\ell, k}\right|+\sum_{\ell=\ell^{*}}^{k}\left|\left(\Lambda^{\ell}-\mathbf{1} r^{T}\right) \otimes M_{\ell, k}\right| \\
& =\sum_{\ell=0}^{\ell^{*}-1}\left|\Lambda^{\ell}-\mathbf{1} r^{T}\right|\left|M_{\ell, k}\right|+\sum_{\ell=\ell^{*}}^{k}\left|\Lambda^{\ell}-\mathbf{1} r^{T}\right|\left|M_{\ell, k}\right| \\
& \leq c \sum_{\ell=0}^{\ell^{*}-1}\left|M_{\ell, k}\right|+\sum_{\ell=\ell^{*}}^{k}\left|\Lambda^{\ell}-\mathbf{1} r^{T}\right| \\
& \leq c \sum_{\ell=0}^{\ell^{*}-1}\left(\begin{array}{l}
k \\
\ell
\end{array}\right) \alpha^{\left\lfloor\frac{k+1}{n}\right\rfloor-\ell}+\sum_{\ell=\ell^{*}}^{k} c \sigma^{\ell} \\
& \leq c \frac{\delta}{2 c}+\frac{\delta}{2} \\
& =\delta
\end{aligned}
$$

where we have employed (12) and (13). Having shown (7), we can write

$$
\begin{aligned}
& \lim _{k \rightarrow \infty}\left|\mathbf{x}(k)-\left(\mathbf{1} r^{T} \otimes Q^{k}\right) \mathbf{x}(0)\right| \\
& =\lim _{k \rightarrow \infty}\left|\left(I_{p} \otimes Q^{k}\right) \mathbf{w}(k)-\left(\mathbf{1} r^{T} \otimes Q^{k}\right) \mathbf{w}(0)\right| \\
& =\lim _{k \rightarrow \infty}\left|\left(I_{p} \otimes Q^{k}\right) \Phi(k, 0) \mathbf{w}(0)-\left(\mathbf{1} r^{T} \otimes Q^{k}\right) \mathbf{w}(0)\right| \\
& =\lim _{k \rightarrow \infty}\left|\left(\left(I_{p} \otimes Q^{k}\right)\left(\mathbf{1} r^{T} \otimes I_{n}\right)-\mathbf{1} r^{T} \otimes Q^{k}\right) \mathbf{w}(0)\right| \\
& =\lim _{k \rightarrow \infty}\left|\left(\mathbf{1} r^{T} \otimes Q^{k}-\mathbf{1} r^{T} \otimes Q^{k}\right) \mathbf{w}(0)\right| \\
& =0
\end{aligned}
$$

where we used the fact that $\mathbf{x}(0)=\mathbf{w}(0)$. Eq. (15) implies

$\lim _{k \rightarrow \infty}\left|\xi_{i}(k)-\left(r^{T} \otimes Q^{k}\right) \mathbf{x}(0)\right|=0$

for all $i \in\{1, \ldots, p\}$. Hence the result.

\section{Synchronization via output feedback}

We are now ready to answer the question asked in the problem statement: Does there exist $L \in \mathbb{R}^{m \times n}$ such that solutions of systems (1) with $u_{i}=L z_{i}$, where $z_{i}$ is as in (2), synchronize for all connected $\Lambda$ ? The answer, we will see, is affirmative and lies in a straightforward generalization of the key result (Theorem 3) of the previous section. We also provide a simple algorithm to calculate such $L$. Let us begin with the following fact.
Fact 4 Let $F \in \mathbb{R}^{n \times n}$ be a neutrally stable matrix with all its eigenvalues having unity magnitude. Then there exists $\mathbb{R}^{n \times n} \ni R=R^{T}>0$ such that $F^{T} R F=R$.

PROOF. Since $F$ has no Jordan block of size greater than one, it can be diagonalized. Therefore there exist $Z \in \mathbb{C}^{n \times n}$ and a diagonal matrix $D \in \mathbb{C}^{n \times n}$ such that $F=Z D Z^{-1}$. Since the diagonal entries of $D$ are all of unity magnitude, $\left|D^{k} x\right|=|x|$ for all $x \in \mathbb{R}^{n}$ and $k=1,2, \ldots$ Therefore there exist real numbers $0<$ $a \leq b<\infty$ such that

$a|x| \leq\left|F^{k} x\right| \leq b|x|, \quad k=1,2, \ldots$

Note that $a^{2} x^{T} x \leq x^{T} F^{k T} F^{k} x \leq b^{2} x^{T} x$ for all $x$ and $k$. Let us define the compact set $\overline{\mathcal{X}}:=\left\{X \in \mathbb{R}^{n \times n}: X=\right.$ $\left.X^{T}, a^{2} I_{n} \leq X \leq b^{2} I_{n}\right\}$ and the continuous function $f: \mathcal{X} \rightarrow \mathbb{R}$ as $f(\bar{X}):=\left|F^{T} X F-X\right|$. Finally let

$X_{k}:=k^{-1} \sum_{i=1}^{k} F^{i T} F^{i}, \quad k=1,2, \ldots$

By construction $a^{2} I_{n} \leq X_{k} \leq b^{2} I_{n}$ and $X_{k}^{T}=X_{k}$ for all $k$. Hence $X_{k} \in \mathcal{X}$. We now can write

$$
\begin{aligned}
f\left(X_{k}\right) & =\left|F^{T} X_{k} F-X_{k}\right| \\
& =k^{-1}\left|\sum_{i=2}^{k+1} F^{i T} F^{i}-\sum_{i=1}^{k} F^{i T} F^{i}\right| \\
& =k^{-1}\left|F^{(k+1) T} F^{k+1}-F^{T} F\right| \\
& \leq k^{-1} 2 b^{2} .
\end{aligned}
$$

As a result we have $\lim _{k \rightarrow \infty} f\left(X_{k}\right)=0$. Since $f$ takes only nonnegative values we deduce

$\inf _{X \in \mathcal{X}} f(X)=0$.

Compactness of $\mathcal{X}$ together with continuity of $f$ implies that minimum is attained [7, Cor. 6.57]. Thus there exists $R \in \mathcal{X}$ such that $f(R)=0$.

Algorithm 1 Given $A \in \mathbb{R}^{n \times n}$ that is neutrally stable and $C \in \mathbb{R}^{m \times n}$, we obtain $L \in \mathbb{R}^{n \times m}$ as follows. Let $n_{1} \leq n$ be the number of eigenvalues of $A$ with unity magnitude. Let $n_{2}:=n-n_{1}$. If $n_{1}=0$, then let $L:=0$; else construct $L$ according to the following steps.

Step 1: Choose $U \in \mathbb{R}^{n \times n_{1}}$ and $W \in \mathbb{R}^{n \times n_{2}}$ satisfying

$$
[U W]^{-1} A[U W]=\left[\begin{array}{cc}
F & 0 \\
0 & G
\end{array}\right]
$$

where all the eigenvalues of $F \in \mathbb{R}^{n_{1} \times n_{1}}$ have unity magnitude. (Assume, without loss of generality for our purposes, that $C U$ is full row rank.) 
Step 2: Choose $R \in \mathbb{R}^{n_{1} \times n_{1}}$ with $R=R^{T}>0$ such that $F^{T} R F=R$. (This we can do thanks to Fact 4.)

Step 3: Choose $H \in \mathbb{R}^{m \times n_{1}}$ satisfying $H H^{T}=I_{m}$ and range $\left(H^{T}\right)=\operatorname{range}\left(R^{-1 / 2} U^{T} C^{T}\right)$. (Note that then $m a-$ trix $C U R^{-1 / 2} H^{T}$ is invertible.)

Step 4: Define $L:=U F R^{-1 / 2} H^{T}\left(C U R^{-1 / 2} H^{T}\right)^{-1}$.

Below is our main result.

Theorem 5 Consider systems (1). Let $u_{i}=L z_{i}$ where $L \in \mathbb{R}^{n \times m}$ is constructed according to Algorithm 1 and $z_{i}$ is as in (2). Then for all network topologies described by connected $\Lambda$, solutions $x_{i}(\cdot)$, for $i=1, \ldots, p$, synchronize to a bounded trajectory (which we explicitly provide in the proof.)

PROOF. Let the variables that are not introduced here be defined as in Algorithm 1. Without loss of generality we assume that $C U$ is full row rank. Since $H^{T} H$ is an orthogonal projection onto $\operatorname{range}\left(H^{T}\right)=\operatorname{range}\left(R^{-1 / 2} U^{T} C^{T}\right)$, we can write $H^{T} H R^{-1 / 2} U^{T} C^{T}=R^{-1 / 2} U^{T} C^{T}$. Taking the transpose we obtain $C U R^{-1 / 2} H^{T} H=C U R^{-1 / 2}$. Since $C U R^{-1 / 2} H^{T}$ is invertible we obtain $H=$ $\left(C U R^{-1 / 2} H^{T}\right)^{-1} C U R^{-1 / 2}$. Therefore $L C U R^{-1 / 2}=$ $U F R^{-1 / 2} H^{T} H$. Also, detectability of $(C, A)$ implies that pair $(H, Q)$ is observable for $Q:=R^{1 / 2} F R^{-1 / 2}$. Note that $Q$ is orthogonal due to $F^{T} R F=R$.

We let $U^{\dagger} \in \mathbb{R}^{n_{1} \times n}$ and $W^{\dagger} \in \mathbb{R}^{n_{2} \times n}$ be such that

$$
\left[\begin{array}{c}
U^{\dagger} \\
W^{\dagger}
\end{array}\right]=\left[\begin{array}{ll}
U & W
\end{array}\right]^{-1}
$$

Note then that $U^{\dagger} U=I_{n_{1}}, W^{\dagger} W=I_{n_{2}}, U^{\dagger} W=0$, and $W^{\dagger} U=0$. Since $u_{i}=L z_{i}$, we can combine (1) and (2) to obtain $x_{i}^{+}=A x_{i}+L C \sum_{j=1}^{p} \lambda_{i j}\left(x_{j}-x_{i}\right)$. By change of variables $\xi_{i}:=\left[\begin{array}{ll}R^{1 / 2} & 0\end{array}\right]\left[\begin{array}{ll}U & W\end{array}\right]^{-1} x_{i}$ and $\eta_{i}:=\left[\begin{array}{ll}0 & I_{n_{2}}\end{array}\right]\left[\begin{array}{ll}U & W\end{array}\right]^{-1} x_{i}$ we can write

$$
\begin{aligned}
\xi_{i}^{+}=Q \xi_{i}+Q & H^{T} H \sum_{j=1}^{p} \lambda_{i j}\left(\xi_{j}-\xi_{i}\right) \\
& +R^{1 / 2} U^{\dagger} L C W \sum_{j=1}^{p} \lambda_{i j}\left(\eta_{j}-\eta_{i}\right)
\end{aligned}
$$

$\eta_{i}^{+}=G \eta_{i}$

Let $\Lambda$ be connected and $r \in \mathbb{R}^{p}$ be such that $\lim _{k \rightarrow \infty} \Lambda^{k}=\mathbf{1} r^{T}$. Then define $\omega_{i}: \mathbb{N} \rightarrow \mathbb{R}^{n_{1}}$ as $\omega_{i}(k):=Q^{-k} \xi_{i}(k)$ and $i=1, \ldots, p$. Let $\mathbf{w}:=$
$\left[\begin{array}{lll}\omega_{1}^{T} & \ldots & \omega_{p}^{T}\end{array}\right]^{T}$ and $\mathbf{v}:=\left[\begin{array}{lll}\eta_{1}^{T} & \ldots & \eta_{p}^{T}\end{array}\right]^{T}$. Starting from (16) and (17) we can write

$$
\begin{array}{r}
\left.\mathbf{w}(k+1)=\left(I_{p n_{1}}+\left(\Lambda-I_{p}\right) \otimes Q^{-k} H^{T} H Q^{k}\right)\right) \mathbf{w}(k) \\
+\left(\left(\Lambda-I_{p}\right) \otimes Q^{-k-1} M G^{k}\right) \mathbf{v}(0)
\end{array}
$$

where $M:=R^{1 / 2} U^{\dagger} L C W$. Thence

$$
\begin{aligned}
& \mathbf{w}(k)=\Phi(k, 0) \mathbf{w}(0) \\
& +\left[\sum_{\ell=0}^{k-1} \Phi(k, \ell+1)\left(\left(\Lambda-I_{p}\right) \otimes Q^{-\ell-1} M G^{\ell}\right)\right] \mathbf{v}(0)
\end{aligned}
$$

where

$\Phi(k, \ell):=\prod_{\tau=\ell}^{k-1}\left(I_{p n_{1}}+\left(\Lambda-I_{p}\right) \otimes Q^{-\tau} H^{T} H Q^{\tau}\right)$

is the state transition matrix [2]. From Theorem 3 we can deduce that $\Phi(k, \ell)$ is uniformly bounded for all $k$ and $\ell$. Also, for any fixed $\ell$ we have $\lim _{k \rightarrow \infty} \Phi(k, \ell)=\mathbf{1} r^{T} \otimes I_{n_{1}}$. Moreover, $Q^{k}$ is uniformly bounded for all $k$, and $G^{k}$ decays exponentially as $t \rightarrow \infty$ for all the eigenvalues of $G$ are strictly within the unit circle. Therefore we can write

$$
\begin{aligned}
& \lim _{k \rightarrow \infty} \sum_{\ell=0}^{k-1} \Phi(k, \ell+1)\left(\left(\Lambda-I_{p}\right) \otimes Q^{-\ell-1} M G^{\ell}\right) \\
& =\sum_{\ell=0}^{\infty}\left(\lim _{k \rightarrow \infty} \Phi(k, \ell+1)\right)\left(\left(\Lambda-I_{p}\right) \otimes Q^{-\ell-1} M G^{\ell}\right) \\
& =\sum_{\ell=0}^{\infty}\left(\mathbf{1} r^{T} \otimes I_{n_{1}}\right)\left(\left(\Lambda-I_{p}\right) \otimes Q^{-\ell-1} M G^{\ell}\right) \\
& =0
\end{aligned}
$$

Then, by (18), we can write

$\lim _{k \rightarrow \infty} \mathbf{w}(k)=\left(\mathbf{1} r^{T} \otimes I_{n_{1}}\right) \mathbf{w}(0)$.

Therefore solutions $\xi_{i}(\cdot)$ synchronize to $\left(r^{T} \otimes Q^{k}\right) \mathbf{w}(0)$. Moreover, $\lim _{k \rightarrow \infty} \mathbf{v}(k)=0$ for $G^{k}$ is exponentially decaying as $k \rightarrow \infty$. In other words, solutions $\eta_{i}(\cdot)$ synchronize to 0 . Now let $\widehat{Q} \in \mathbb{R}^{n \times n}$ and $Z \in \mathbb{R}^{n \times n}$ be

$\widehat{Q}:=\left[\begin{array}{cc}Q & 0 \\ 0 & 0\end{array}\right], \quad Z:=\left[\begin{array}{c}R^{1 / 2} U^{\dagger} \\ W^{\dagger}\end{array}\right]$

Then we can conclude that solutions $x_{i}(\cdot)$ synchronize to

$\bar{x}(k):=\left(r^{T} \otimes Z^{-1} \widehat{Q}^{k} Z\right)\left[\begin{array}{c}x_{1}(0) \\ \vdots \\ x_{p}(0)\end{array}\right]$ 
Hence the result.

\section{References}

[1] D. Angeli and P.-A. Bliman. Stability of leaderless discretetime multi-agent systems. Mathematics of Control, Signals ES Systems, 18:293-322, 2006.

[2] P.J. Antsaklis and A.N. Michel. Linear Systems. McGrawHill, 1997.

[3] M. Arcak. Passivity as a design tool for group coordination. IEEE Transactions on Automatic Control, 52:1380-1390, 2007.

[4] I. Belykh, V. Belykh, and M. Hasler. Generalized connection graph method for synchronization in asymmetrical networks. Physica D, 224:42-51, 2006.

[5] V.D. Blondel, J.M. Hendrickx, A. Olshevsky, and J.N. Tsitsiklis. Convergence in multiagent coordination, consensus, and flocking. In Proc. of the 44 th IEEE Conference on Decision and Control, pages 2996-3000, 2005.

[6] S. Boccaletti, V. Latora, Y. Moreno, M. Chavez, and D.U. Hwang. Complex networks: structure and dynamics. Physics Reports-Review Section of Physics Letters, 424:175308, 2006.

[7] A. Browder. Mathematical Analysis: An Introduction. Springer, 1996.

[8] C. Cai and G. Chen. Synchronization of complex dynamical networks by the incremental ISS approach. Phyisica A, 371:754-766, 2006.

[9] Q. Hui, W.M. Haddad, and S.P. Bhat. Finite-time semistability theory with applications to consensus protocols in dynamical networks. In Proc. of the 2007 American Control Conference, pages 2411-2416, 2007.

[10] A. Jadbabaie, J. Lin, and A.S. Morse. Coordination of groups of mobile autonomous agents using nearest neighbor rules. IEEE Transactions on Automatic Control, 48:9881001, 2003.

[11] L. Moreau. Stability of multi-agent systems with timedependent communication links. IEEE Transactions on Automatic Control, 50:169-182, 2005.

[12] R. Olfati-Saber, J.A. Fax, and R.M. Murray. Consensus and cooperation in networked multi-agent systems. Proceedings of the IEEE, 95:215-233, 2007.

[13] R. Olfati-Saber and R.M. Murray. Consensus problems in networks of agents with switching topology and time-delays. IEEE Transactions on Automatic Control, 49(9):1520-1533, 2004.

[14] L.M. Pecora and T.L. Carroll. Master stability functions for synchronized coupled systems. Physical Review Letters, 80:2109-2112, 1998.

[15] W. Ren and R.W. Beard. Consensus seeking in multiagent systems under dynamically changing interaction topologies. IEEE Transactions on Automatic Control, 50:655-661, 2005.

[16] J.J.E. Slotine, W. Wang, and K. El-Rifai. Contraction analysis of synchronization in networks of nonlinearly coupled oscillators. In Proc. of the 16th International Symposium on Mathematical Theory of Networks and Systems, Belgium, 2004.

[17] G.-B. Stan and R. Sepulchre. Analysis of interconnected oscillators by dissipativity theory. IEEE Transactions on Automatic Control, 52:256-270, 2007.
[18] S.H. Strogatz. Exploring complex networks. Nature, 410:268276, 2001.

[19] J.N. Tsitsiklis, D.P. Bertsekas, and M. Athans. Distributed asynchronous deterministic and stochastic gradient optimization algorithms. IEEE Transactions on Automatic Control, 31:803-812, 1986.

[20] S.E. Tuna and R. Sepulchre. Quantitative convergence analysis of multi-agent systems. In Proc. of the 7th IFAC Symposium on Nonlinear Control Systems, pages 236-241, 2007.

[21] X.F. Wang. Complex networks: topology, dynamics and synchronization. International Journal of Bifurcation and Chaos, 12:885-916, 2002.

[22] C.W. Wu and L.O. Chua. Synchronization in an array of linearly coupled dynamical systems. IEEE Transactions on Circuits and Systems-I, 42:430447, 1995. 